Service social

\title{
La sécurité alimentaire à l’agenda politique québécois
}

\section{Anne-Marie Hamelin et Nathalie Bolduc}

Volume 50, numéro 1, 2003

URI : https://id.erudit.org/iderudit/006919ar

DOI : https://doi.org/10.7202/006919ar

Aller au sommaire du numéro

Éditeur(s)

École de service social de l'Université Laval

ISSN

1708-1734 (numérique)

Découvrir la revue

Citer cet article

Hamelin, A.-M. \& Bolduc, N. (2003). La sécurité alimentaire à l'agenda politique québécois. Service social, 50(1), 57-80. https://doi.org/10.7202/006919ar

\section{Résumé de l'article}

Au printemps 2001, la lutte contre la pauvreté et l'exclusion sociale figure au titre des défis majeurs du Québec dans la poursuite de son développement social et économique. Le Discours sur le budget en fait foi : 815 millions de dollars sont alors consentis sur trois ans à cette lutte, dont 100 millions en vue d'investissements structurants devant favoriser l'émergence de nouvelles initiatives de solidarité sociale. Dans cette foulée, une somme de 10 millions était octroyée pour la mise en oeuvre de projets de sécurité alimentaire visant à améliorer l'autonomie des familles. Les auteures voient dans cette mesure, même modeste, une ouverture significative à l'égard de la sécurité alimentaire. Qui plus est, ce thème s'inscrit en 2002 dans la loi visant à lutter contre la pauvreté et l'exclusion sociale. Les auteures proposent comme explication à cette ouverture un " effet de contagion » qui a pu se réaliser pleinement par l'action convergente de trois courants - celui des problèmes, celui des solutions et celui de la politique - et des acteurs qui y ont pris part. 


\title{
La sécurité alimentaire à l'agenda politique québécois ${ }^{1}$
}

\author{
Anne-Marie HAMELIN \\ Professeure au Département \\ des sciences des aliments et de nutrition \\ Université Laval à Québec \\ Courriel : anne-marie.hamelin@aln.ulaval.ca \\ Nathalie BoLDUC \\ Professionnelle de recherche \\ Conseil de la santé et du bien-être à Québec \\ Courriel : nathalie.bolduc@msss.gouv.qc.ca
}

Au printemps 2001, la lutte contre la pauvreté et l'exclusion sociale figure au titre des défis majeurs du Québec dans la poursuite de son développement social et économique. Le Discours sur le budget en fait foi : 815 millions de dollars sont alors consentis sur trois ans à cette lutte, dont 100 millions en vue d'investissements structurants devant favoriser l'émergence de nouvelles initiatives de solidarité sociale. Dans cette foulée, une somme de 10 millions était octroyée pour la mise en œuvre de projets de sécurité alimentaire visant à améliorer l'autonomie des familles. Les auteures voient dans cette mesure, même modeste, une ouverture significative à l'égard de la sécurité alimentaire. Qui plus est, ce thème s'inscrit en 2002 dans la loi visant à lutter contre la pauvreté et l'exclusion sociale. Les auteures proposent comme explication à cette ouverture un " effet de contagion » qui a pu se réaliser pleinement par l'action convergente de trois courants - celui des problèmes, celui des solutions et celui de la politique - et des acteurs qui y ont pris part.

Mots clés : sécurité alimentaire des ménages, lutte contre la pauvreté, politique publique, action politique, effet de contagion.

1. Ce texte s'inspire d'une communication présentée par A.M.Hamelin au Congrès annuel des diététistes du Canada à St. John (N.-B.), le 8 juin 2002, et intitulée How Food Security Got to Be an Issue on the Governmental Agenda in Québec. 
In the spring 2001, the fight against poverty and social exclusion appears one of the major priorities of the Government of Québec in the pursuit of its social and economic development. $815 \mathrm{M} \$$ over a three-year period were thus allocated for this fight, including a $100 \mathrm{M} \$$ social solidarity reserve to fund formative investments geared toward new initiatives of social solidarity. Among these investments, $10 \mathrm{M} \$$ were allocated for the implementation of food security projects aimed at enhancing family food autonomy. Despite the modesty of this investment, the authors see in this announcement a significant opening toward food security. Moreover, in 2002, food security becomes entrenched in an act to combat poverty and social exclusion. As an explanation to this opening, this article proposes a "spillover" effect that had been fully realised by the coupling of three streams, that of problems, solutions, and politics, and the influence of policy entrepreneurs.

Key words: household food security, fight against poverty, public policy, political action, spillover.

\section{INTRODUCTION}

Les problèmes publics portés à l'attention des décideurs politiques sont nombreux. Toutefois, seuls quelques-uns font l'objet d'une intervention politique, car ils doivent a priori être inscrits à l'agenda gouvernemental. Par conséquent, la mise à l'étude d'un problème est une étape cruciale dans le processus d'émergence d'une politique publique (Cobb et Elder, 1983; Kingdon, 1995; Portz, 1996; Soroka, 2002).

Le modèle de Kingdon (1995) tenant lieu de cadre théorique, la présente étude poursuit deux objectifs : 1) décrire le cheminement par lequel la sécurité alimentaire est parvenue à l'agenda gouvernemental et 2) fournir des éléments d'explication dans la progression du dossier jusqu'à la décision politique. À cette fin, une revue documentaire, complétée par deux entrevues semi-structurées menées auprès de personnes significatives dans l'avancement du dossier, a été réalisée.

Le concept de sécurité alimentaire renvoie à un état selon lequel les populations ont accès en tout temps, dans la dignité, à suffisamment d'aliments pour pouvoir mener une vie saine et active 
(Anderson, 1990; Radimer et al., 1992). II dépasse donc la seule satisfaction des besoins nutritionnels (Campbell, 1991) et évoque un état relatif d'autonomie alimentaire ${ }^{2}$. Il y a insécurité alimentaire lorsque la disponibilité d'aliments sains et nutritionnellement adéquats, ou la capacité d'acquérir des aliments personnellement satisfaisants par des moyens socialement acceptables, est limitée ou incertaine (Anderson, 1990; Radimer et al., 1992). Dans le Québec méridional, il s'agit surtout d'un problème d'accès, et non de disponibilité. Ainsi, environ $8 \%$ de la population de 15 ans ou plus rapportait souffrir d'au moins un aspect d'insécurité alimentaire parmi ceux mesurés dans l'Enquête sociale et de santé 1998 (monotonie du régime; restriction de l'apport alimentaire; incapacité d'offrir des repas équilibrés aux enfants); la proportion s'élevait à $39 \%$ chez les personnes vivant dans des ménages très pauvres (Institut de la statistique du Québec, 2000). Considérant que seules trois facettes de l'expérience ont été sondées, ces données sous-estiment probablement la prévalence réelle d'insécurité alimentaire des ménages dans la population québécoise, celle-ci oscillant autour de $10 \%$ au Canada (Statistique Canada, 2001) et aux États-Unis (USDA, 2000). L'insécurité alimentaire est associée au phénomène de la pauvreté.

Pour comprendre comment la question de la sécurité alimentaire s'est retrouvée inscrite à l'agenda, il nous faut revenir une dizaine d'années en arrière.

2. En ce sens, le dépannage alimentaire (p. ex. : recevoir un colis de nourriture) et les autres formes de soutien alimentaire ne sauraient en aucun cas constituer la sécurité alimentaire, même s'ils pourraient, dans certaines circonstances et de façon ponctuelle, y contribuer. 


\section{LES PRÉOCCUPATIONS DU QUÉBEC À L'ENDROIT DE L'INSÉCURITÉ ALIMENTAIRE DES FAMILLES}

\section{D'une alimentation insuffisante...}

Les préoccupations du Québec à l'endroit de l'insécurité alimentaire des familles remontent au début des années $1990^{3}$; on parle alors d' " une alimentation quantitativement et qualitativement insuffisante » (ministère de la Santé et des Services sociaux, 1993, p. 15). La Politique de la santé et du bien-être (1992) reconnaît que le revenu dont disposent certaines familles ne leur permet pas toujours de manger suffisamment chaque jour; elle souligne aussi le nombre croissant d'enfants qui ont faim et entend lutter contre ce problème (p. 139). Outre le ministère de la Santé et des Services sociaux (MSSS), la question de «l'accessibilité socioéconomique et physique " aux aliments interpelle aussi, à divers titres, d'autres instances. Ainsi, le ministère de l'Éducation (MEQ), dans le cadre du plan d'action sur la réussite éducative, gère une mesure de soutien alimentaire (distribution de petits déjeuners, de collations ou de repas le midi) destinée aux écoliers des milieux défavorisés de l'île de Montréal et de Québec, selon les besoins déterminés par les commissions scolaires. Le MEQ assume en outre depuis décembre 1992 la direction et l'exécution du Programme de distribution de lait gratuit dans les écoles primaires du Québec, dont l'application est restreinte aux milieux les plus défavorisés; la responsabilité du Programme incombait jusque-là au ministère de l'Agriculture, des Pêcheries et de l'Alimentation. Le ministère de la Main-d'œuvre, de la Sécurité du revenu et de la Formation professionnelle s'intéresse pour sa part à la problématique de l'alimentation des prestataires de la sécurité du revenu et s'apprête en 1994 à mettre sur pied un projet de soutien à l'alimentation des familles monoparentales. Le projet qui s'inscrit dans une approche globale d'intégration sociale et professionnelle des prestataires est axé sur l'acquisition de connaissances et

3. Déjà depuis les années 1970 le Québec est sensible à la situation alimentaire des familles dans les milieux défavorisés, comme en témoignent, entre autres, la mise en œuvre de divers programmes de soutien alimentaire aux femmes enceintes (p. ex. : programme OLO, « œufs-lait-oranges »), aux écoliers (plan Pagé), de même que la longue série d'engagements et de débats autour de la distribution de lait gratuit dans les écoles primaires. 
d'habitudes plus saines liées au revenu disponible (Lemieux, 1995). Le MAPAQ, partenaire de première heure au Comité intersectoriel de nutrition, a quant à lui une vision de la sécurité alimentaire tournée vers la disponibilité alimentaire. En 1993, ces quatre ministères ainsi que les directions régionales de la santé publique et des partenaires de divers secteurs (scolaire, communautaire, industriel, médiatique, parapublic et professionnel) sont réunis par le directeur de la Promotion de la santé et du bien-être en vue de la création d'un partenariat intersectoriel pour une saine alimentation au Québec; « ... l'accès à une alimentation suffisante en qualité et en quantité... » (p. 11) est une préoccupation partagée à divers degrés par les participants (ministère de la Santé et des Services sociaux, 1993).

\section{Vers le besoin de bâtir la sécurité alimentaire}

Témoin de la précarité économique des familles, le secteur communautaire est particulièrement sensible à la question, lui qui, dans l'ombre, œuvre depuis bon nombre d'années à soulager les familles de la faim ${ }^{4}$. Au début des années 1990, les banques alimentaires et les organismes de dépannage alimentaire d'urgence se multiplient ${ }^{5}$; le réseau d'aide alimentaire s'organise dans plusieurs régions du Québec. L'augmentation apparente du nombre d'usagers au cours des années force la réflexion sur le sens et le rôle de l'aide alimentaire. Le réseau est soutenu dans son questionnement par les tables régionales de concertation ( $p$. ex. : Table de concertation sur la faim du Montréal métropolitain, 1992) et par le milieu de la recherche (p. ex. : Côté, Racine et Rouffignat, 1995; Beeman, Panet-Raymond et Rouffignat, 1996). Le comité multisectoriel «Nourrir Montréal », né sous l'égide de «Vivre Montréal en santé » en 1990, développe une vision élargie de

4. Par exemple en 1973, à Pointe-Saint-Charles - quartier défavorisé du Centre Sud-Ouest de Montréal -, le Carrefour d'éducation populaire mettait sur pied un atelier d'éducation à la consommation et à la nutrition «ActionAlimentation ». La première banque alimentaire au Québec, Moisson-Montréal, a vu le jour en 1984.

5. A titre d'exemple, voir les multiples ressources communautaires ou privées de l'île de Montréal et ses environs dans le Répertoire des organismes d'aide alimentaire du Montréal métropolitain (Table de concertation sur la faim du Montréal métropolitain, 1993). 
lutte contre la faim et, dans une recherche de solution à plus long terme, s'intéresse aux causes de l'insécurité alimentaire (Bertrand et Dupont, 1998). Cette approche rejoint celle de l'Association canadienne de la Journée mondiale de l'alimentation (ACJMA) qui, fidèle à son plan stratégique, stimule l'action collective pour assurer la sécurité alimentaire ici et ailleurs (Association canadienne de la Journée mondiale de l'alimentation, 1992). L'action communautaire autour de la sécurité alimentaire entre dans une période de pleine effervescence. Le colloque «Au-delà du dépannage alimentaire ", organisé par le Comité Québec de l'ACJMA et tenu au Château Frontenac en 1992, est l'occasion pour les participants de réfléchir au concept de sécurité alimentaire proposé par les Nations Unies (United Nations, 1991). L'événement est aussi l'occasion de discuter des mérites et des limites du dépannage alimentaire, de même que de la portée des actions dites alternatives au dépannage (p. ex. : cuisines collectives; ateliers d'éducation à la consommation et à la nutrition; clubs de consommateurs) (Dufour, 1992). À cet égard, ce colloque représente un jalon dans la compréhension des enjeux et donne le ton aux colloques qui vont suivre (p. ex. : Table d'interaction sur la faim de Québec, 1994). II contribue aussi à inscrire l'expression " sécurité alimentaire » dans le langage. Déjà, en 1993, le secteur communautaire presse le gouvernement de "développer des politiques sociales et économiques afin d'assurer la "sécurité alimentaire" pour tous " (ministère de la Santé et des Services sociaux, 1993, p. 45). Le terme est aussi récupéré dans les démarches régionales. Par exemple, en 1995 à Montréal, à la suite d'un exercice de "priorisation » régionale des objectifs de santé, la Régie régionale de la santé et des services sociaux de MontréalCentre décide d'accorder des crédits de $120000 \$$ pour l'élaboration d'un projet régional de sécurité alimentaire avec le concours entre autres du Conseil régional de développement (Bertrand et Dupont, 1998). C'est la première fois qu'au Québec on investit un montant de cet ordre dans la sécurité alimentaire proprement dite. Le sillon est tracé. La sécurité alimentaire fait l'objet d'un avis de la part de l'Ordre professionnel des diététistes du Québec (Leduc-Gauvin, Cossette, Lépine et Malette, 1996). La réflexion se poursuit au sein des tables de concertation et à l'occasion de divers colloques (p. ex. : Table de concertation sur la faim du Montréal métropolitain, 1996; Delisle et Hamelin, 1997). 
Ces travaux et réalisations de la société civile, du milieu professionnel, de la recherche et du parapublic amènent peu à peu le MSSS à s'interroger sur l'essor des banques alimentaires dont les activités représentent alors le principal indicateur du phénomène d'insécurité alimentaire (indicateur indirect).

\section{Le besoin entendu}

Ce n'est qu'à la fin des années 1990 que le développement de connaissances plus formelles sur l'expérience globale d'insécurité alimentaire (Hamelin, 1999) permet de valider, au Québec, une première génération d'indicateurs directs existant aux États-Unis (Radimer et al., 1992). Quelques-uns des énoncés ${ }^{6}$ du questionnaire Radimer/Cornell servent alors à constituer un indice d'insécurité alimentaire qui sera incorporé dans l'Enquête sociale et de santé 1998 (Institut de la statistique du Québec, 2000). Les résultats de l'enquête viennent "corroborer », à l'échelle de la population, le vécu d'insécurité alimentaire des ménages, de même que son lien avec la pauvreté : la présence de l'une ou l'autre des manifestations d'insécurité alimentaire, parmi celles étudiées, est en effet plus souvent observée parmi les personnes sans emploi $(27 \%)$ et les personnes vivant dans des ménages très pauvres (39\%) ou pauvres (20\%) (Institut de la statistique du Québec, 2000). Des données qui appuient celles des organismes communautaires faisant de l'aide alimentaire (Rouffignat, Racine et Côté, 1996). Par ailleurs, le spectre des répercussions d'insécurité alimentaire sur la santé et la société commence à se préciser (Hamelin, Habicht et Beaudry, 1999).

Tout en continuant à lutter contre la faim par des mesures de soutien alimentaire direct, le gouvernement manifeste publiquement son intérêt pour la sécurité alimentaire des ménages dans la

6. Les trois énoncés du questionnaire Radimer/Cornell (qui en compte treize) retenus dans le cadre de l'Enquête, et auxquels les ménages sont appelés à dire si ces situations sont souvent vraies, parfois vraies ou jamais vraies, sont: 1) Nous mangeons la même chose plusieurs jours de suite parce que nous n'avons que quelques aliments sous la main et n'avons pas d'argent pour en racheter (en acheter d'autres); 2) Nous mangeons moins que nous devrions parce que nous n'avons pas assez d'argent pour la nourriture; 3) Nous ne pouvons pas offrir des repas équilibrés aux enfants de notre foyer parce que nous ne pouvons pas nous le permettre financièrement. 
seconde moitié des années 1990, comme en témoigne un discours prononcé par la sous-ministre adjointe à la Santé et aux Services sociaux (Colin, 1997). Lors du lancement du Mois de la nutrition 1997, celle-ci a réitéré l'engagement ministériel récent d'intensifier l'intervention dans la lutte contre l'insécurité alimentaire. Quatre axes d'intervention sont ciblés: le renforcement de l'action intersectorielle avec les autres secteurs d'activité gouvernementale (notamment ceux de l'Emploi et de la Solidarité, de l'Éducation ainsi que de l'Agriculture, des Pêcheries et de l'Alimentation) et les directions régionales de santé publique; le soutien financier aux organismes communautaires; la mise en œuvre de projets pilotes sous la responsabilité des régies régionales; et le développement de la recherche sur le thème de la sécurité alimentaire (Colin, 1997). Cette même année, le Ministère, par l'entremise de la Direction de la santé publique, accorde aux régions sociosanitaires du Québec un budget non récurrent de 600000 \$ en vue de favoriser l'émergence d'une préoccupation pour la concertation des actions relatives à la sécurité alimentaire. L'intérêt porte de plus en plus sur la sécurité alimentaire en tant que condition pouvant contribuer à atténuer les conséquences de la pauvreté. Aussi la Direction générale de la santé publique du MSSS, conjointement avec les régies régionales de la santé et des services sociaux, vise-t-elle, dans le contexte de la lutte aux inégalités en matière de santé et de bien-être (ministère de la Santé et des Services sociaux, 1997), à mettre en place un ensemble de stratégies et d'actions qui contribuent à la sécurité alimentaire de la population du Québec. Dans le cadre des actions concertées du Conseil québécois de la recherche sociale (CQRS), le MSSS lance, en 1998, un appel d'offres afin de déterminer l'impact des pratiques en matière de sécurité alimentaire sur les personnes et les collectivités. À ce moment-là, les pratiques dites alternatives ( $p$. ex. : cuisines collectives) au dépannage alimentaire sont perçues comme des actions de sécurité alimentaire (voir Les partenaires pour le développement de la sécurité alimentaire, 2000). Par ailleurs, la sensibilisation du gouvernement au thème de la sécurité alimentaire se poursuit par d'autres voies, dont celle des demandes de financement adressées par les organismes communautaires à ses diverses instances ( $p$. ex. : Secrétariat à l'action communautaire autonome du Québec). 


\section{PRIORITÉ À LA LUTTE CONTRE LA PAUVRETÉ}

\section{Un contexte favorable}

Des années de lutte au déficit viennent sérieusement aggraver l'état de pauvreté, une situation largement dénoncée par divers groupes sociaux et syndicaux au fil des années 1990. La marche Du pain et des roses (Fédération des femmes du Québec, 1995), puis le manifeste du $1^{\mathrm{er}}$ mai de l'an 2000 « Une société sans pauvreté ", signé par les centrales syndicales du Québec, le Collectif pour une loi sur l'élimination de la pauvreté et la Fédération des femmes du Québec, envoient un signal au gouvernement. Au printemps 2000, le Conseil des ministres confie au ministre de la Solidarité sociale, de concert avec le Secrétaire général du Conseil exécutif, le soin de proposer au gouvernement une stratégie de lutte contre la pauvreté. Les autres ministères et les organismes directement appelés à collaborer à l'élaboration de la stratégie sont: Santé et Services sociaux, Éducation, Famille et Enfance, Finances, Relations avec les citoyens et Immigration, Société d'habitation du Québec, Régie des rentes et Institut de la statistique du Québec; parmi eux, certains, comme le MSSS et le $M E Q$, sont déjà sensibilisés au problème d'insécurité alimentaire. Pour sa part, l'Institut de la statistique du Québec vient d'introduire pour la première fois une mesure d'insécurité alimentaire dans l'Enquête sociale et de santé 1998.

Parallèlement, un changement est en train de s'opérer dans la compréhension gouvernementale du phénomène de la pauvreté. Le Forum sur le développement social, organisé quelques années plus tôt par le Conseil de la santé et du bien-être en collaboration avec un ensemble de partenaires locaux et régionaux, avait recueilli un large consensus autour de l'idée que la pauvreté est plus qu'une question de revenu : la pauvreté revêt sans contredit une dimension sociale importante dont il faut tenir compte (Conseil de la santé et du bien-être, 1998, p. 10). C'est une idée que partagent également de nombreux chercheurs, dont certains du domaine de la sécurité alimentaire (p. ex. : Sabourin, Hurtubise et Lacourse, 2000). L'idée fait son chemin... Sous l'action répétée de groupes de pression (p. ex.: Collectif pour une loi sur l'élimination de la pauvreté; Fédération des femmes du Québec; 
Front commun des personnes assistées sociales du Québec; Front d'action populaire en réaménagement urbain), le gouvernement constate que les mesures " traditionnelles » de lutte contre le chômage et de valorisation du travail ne peuvent, telles qu'elles existent, enrayer à elles seules la pauvreté. Afin de lutter plus efficacement contre celle-ci, le gouvernement cherchera dorénavant à créer une synergie entre le développement économique et le développement social. Au printemps 2001, la lutte contre la pauvreté et l'exclusion sociale figure au titre des défis majeurs à relever par le Québec dans la poursuite de son développement. Le gouvernement du Parti Québécois veut en faire un projet mobilisateur auprès de l'ensemble de la société.

Le moment semble propice pour la question de sécurité alimentaire. Un rapport sur les effets des pratiques en cette matière dans les régions du Québec est sur le point d'être déposé en réponse à l'appel du MSSS. La question jusqu'alors véhiculée au gouvernement principalement par la voix du $\mathrm{MSSS}^{7}$ intéresse le ministère de l'Emploi et de la Solidarité sociale (MESS), qui est en pleine délibération sur les orientations et les perspectives d'action en matière de lutte contre la pauvreté. Dans cet effort de concertation ministérielle, l'arrivée de personnes auparavant parties prenantes au dossier de la sécurité alimentaire au MSSS tombe à point et renouvelle l'intérêt pour la question. D'entrée de jeu, ces personnes font part des connaissances qu'elles ont acquises au MSSS à l'occasion de divers colloques et entretiens au cours desquels la relation entre l'insécurité alimentaire et la pauvreté ainsi que la pertinence des moyens d'intervention actuels ont été discutées (p. ex. : journée de réflexion sur la question à la Régie régionale de la santé et des services sociaux de la Montérégie, le 13 mars 2000). Sensibilisées à la question, elles trouvent dans leurs nouvelles fonctions à la Direction générale adjointe des politiques de sécurité du revenu l'occasion de faire progresser le dossier.

L'élaboration de la stratégie gouvernementale de lutte contre la pauvreté poursuit son cours. Dans le cadre de ses travaux, le MESS mettait sur pied, au printemps 2001, un comité consultatif

7. Le ministère de l'Agriculture, des Pêcheries et de l'Alimentation, qui a une vocation économique, s'intéresse aussi à la sécurité alimentaire, mais principalement du point de vue de la production et de l'innocuité des aliments. 
(groupe d'experts ${ }^{8}$ ) dont le mandat consiste à fournir un avis au ministre sur les orientations liées à la stratégie, de même qu'à apporter l'éclairage scientifique ou pratique nécessaire à l'évolution des travaux. L'expertise des membres du comité est mise à contribution afin d'aider le Ministère à prévenir la pauvreté et à améliorer les conditions de vie des personnes défavorisées. Les voies novatrices sont encouragées.

\section{Plaidoyer en faveur de la sécurité alimentaire}

En avril 2001, l'un des membres du comité, invité à titre personnel (scientifique) à soumettre des propositions d'action en matière de lutte à la pauvreté, y va d'un " plaidoyer » en faveur de la sécurité alimentaire. Après des commentaires introductifs sur la sécurité alimentaire où les caractéristiques essentielles du phénomène au Québec sont exposées, le propos est centré sur deux idées maîtresses: 1) les limites de l'action communautaire dans la recherche de solutions à la faim et 2) la place incontournable de la sécurité alimentaire dans une stratégie globale de lutte à la pau-vreté et de développement social. Les arguments présentés se résument ainsi. Quant aux limites précitées, l'étude de la correspondance entre la nature du problème et les interventions dominantes actuelles (aide alimentaire surtout) laisse d'abord supposer que celles-ci sont nettement insuffisantes pour éliminer les conditions initiales, puis espérer un changement à long terme dans la situation de sécurité alimentaire des ménages québécois. Bien que les initiatives locales actuelles soient indispensables à court terme pour soulager de la faim les ménages, et que certaines d'entre elles pourraient possiblement contribuer à améliorer la capacité des ménages à faire face aux risques, elles ne permettent pas de prévenir l'insécurité alimentaire ni de bâtir une sécurité alimentaire durable. II est peu probable que l'insécurité alimentaire soit renversée tant qu'on ne mettra pas en œuvre des interventions à moyen et à long terme orientées spécifiquement vers les conditions de risque structurelles (un revenu insuffisant, par exemple) et contextuelles à l'insécurité alimentaire (manque de

8. Composé d'universitaires des domaines de la santé, de la sociologie, de l'économique et du développement communautaire ainsi que de hauts fonctionnaires du MESS. 
logements à prix modique; règles administratives qui n'encouragent pas l'épargne; incitation à la surconsommation). Le problème n'étant pas tant le manque d'aliments que le processus sociétal qui mène au manque d'aliments et à l'absence de contrôle des ménages ou des individus sur leur sécurité alimentaire. II est nécessaire d'intervenir simultanément aux différents niveaux des causes, tout en coordonnant les efforts de tous les partenaires.

Il apparaît en outre qu'une stratégie de lutte à la pauvreté doit prendre en considération le besoin de sécurité alimentaire et lui réserver une place spécifique pour les raisons suivantes:

- la sécurité alimentaire est un enjeu intersectoriel incontournable dans une perspective de développement durable;

- elle a un impact direct sur le sentiment de dignité des familles et des individus;

- elle exerce une influence directe sur le quotidien des familles ("C'est toute la vie qui est bouleversée quand il n'y a plus d'aliments sur la table »);

- elle est susceptible de favoriser l'autonomie (capacité de se prendre en charge) et la participation sociale des groupes qui sont les plus touchés par la pauvreté;

- elle est susceptible de favoriser l'égalité des chances en offrant à tous la possibilité de combler un besoin primaire (celui de se nourrir adéquatement) et de développer leur potentiel (la sécurité alimentaire contribuerait à accroître la capacité de concentration et d'effort soutenu chez les adultes comme chez les enfants);

- elle est susceptible de faire reculer la pauvreté. À cet effet, l'hypothèse d'un lien bidirectionnel entre sécurité alimentaire et pauvreté est émise. Les membres du comité sont invités à voir la sécurité alimentaire comme pouvant contribuer à la réduction de la pauvreté au lieu de seulement considérer l'insécurité alimentaire en tant que conséquence de la pauvreté que l'on cherche à atténuer.

Enfin, l'idée que la sécurité alimentaire devienne un indicateur de progrès est évoquée. Par les dimensions sociales et culturelles qu'il comporte, un tel indicateur permettrait de rendre compte, du 
moins en partie, des effets des solutions mises en œuvre par le gouvernement relativement à la santé et au bien-être de la population.

Ce «plaidoyer » est bien accueilli par les membres du comité. II semble avoir fait son effet : la question, jusqu'alors pressentie par le MESS comme pouvant offrir un intérêt certain, est validée dans sa portée potentielle. La sécurité alimentaire fait maintenant l'objet d'une considération très sérieuse en tant que levier dans la lutte contre la pauvreté et l'exclusion sociale. Une demande de formulation d'un axe d'intervention en sécurité alimentaire est adressée dans les 24 heures par la Direction générale adjointe des politiques de sécurité du revenu à l'auteure de la proposition.

\section{Annonce d'un investissement}

Au printemps 2001, « une offensive majeure contre la pauvreté et l'exclusion » caractérise le Discours sur le budget (ministère des Finances, 2001, p. 4) : 815 millions de dollars sur une période de trois ans sont alors consentis à cette lutte, dont une réserve de 100 millions en vue d'investissements structurants susceptibles de favoriser l'émergence de nouvelles initiatives de solidarité sociale. En juin de la même année, le premier ministre du Québec, de concert avec le ministre d'État au Travail, à l'Emploi et à la Solidarité sociale et la ministre déléguée à la Lutte contre la pauvreté et l'exclusion, annonce que des moyens seront mis en œuvre pour intensifier l'action en matière de lutte contre la pauvreté. Les orientations et les perspectives d'action envisagées, présentées dans le document $\mathrm{Ne}$ laisser personne de côté, sont soumises à la discussion au moment des tournées régionales concernant l'élaboration de la stratégie nationale de lutte à la pauvreté. Le thème de l'insécurité alimentaire est présent (p. 21).

Parmi les investissements proposés, 34,8 millions de dollars sont attribués pour la satisfaction des besoins en alimentation. De ce montant, 21 millions sont alloués au soutien alimentaire des élèves du secondaire et 3,8 millions vont au Club des petits déjeuners du Québec en continuité avec les investissements antérieurs. En marge de ces mesures de soutien alimentaire direct qui atteignent un montant de 24,8 millions de dollars, une somme de 
10 millions est nouvellement octroyée pour le financement d'initiatives de type alternatif en sécurité alimentaire dans le but d'améliorer l'autonomie des familles quant à leur situation alimentaire. Cette enveloppe budgétaire de 10 millions doit être gérée par les régies régionales de la santé et des services sociaux et leurs partenaires qui s'appuieront sur un cadre de référence élaboré par un groupe de travail mandaté par le ministère de la Santé et des Services sociaux (ministère de la Santé et des Services sociaux, 2002).

Comment les actions et événements évoqués plus haut ont-ils contribué à un tel investissement en sécurité alimentaire?

\section{ÉLÉMENTS D’EXPLICATION}

Dans son ouvrage Agendas, Alternatives and Public Policies, Kingdon (1995) propose un modèle théorique qui permet d'expliquer l'inscription d'une question à l'agenda gouvernemental et le début de la formulation d'une politique. L'examen de plusieurs politiques publiques américaines l'ont amené à constater l'existence de trois courants distincts qui se croisent à l'occasion : le courant des problèmes, le courant des solutions et le courant de la politique. II y a inscription d'une question ou d'une politique à l'agenda lorsqu'une "fenêtre politique » s'ouvre, c'est-à-dire lorsque des conditions favorables à la rencontre des trois courants se présentent.

Une ouverture survient quand un nouveau problème attire l'attention des membres d'un gouvernement ou à la suite d'un changement dans le courant des priorités politiques. Une telle " fenêtre » ne reste jamais longtemps ouverte. L'action des entrepreneurs, nom que donne Kingdon (1995) aux acteurs influents, est à ce moment déterminante, car ceux-ci jouent le rôle de rassembleurs des trois courants. Ces acteurs peuvent être issus autant de l'intérieur de l'appareil gouvernemental (ministres, parlementaires et fonctionnaires) que de l'extérieur (groupes d'intérêts, chercheurs, médias, partis politiques, opinion publique). Dans leurs actions, ils utilisent les ressources dont ils disposent, comme leur temps, leur énergie, leur réputation et leur argent, pour faire la promotion d'une position. Pour réussir dans leurs actions, les 
entrepreneurs doivent disposer d'une certaine audience, soit en raison de leur expertise, de leur habileté en communication ou de leur position dans une organisation. Ils doivent également entretenir des relations politiques ou à tout le moins posséder des habiletés en négociation. La réussite de leur action passe également par la ténacité dont ils feront preuve. Kingdon explique que, dans le courant des problèmes, les entrepreneurs doivent convaincre les décideurs de l'existence de problèmes publics. Par la suite, ils doivent montrer que des options pour résoudre les problèmes publics sont disponibles (courant des solutions). Enfin, des conditions politiques favorables sont nécessaires (courant de la politique). Ce dernier courant est influencé par différents éléments : le climat politique ou la tendance nationale (national mood), l'action des groupes d'intérêts, les changements de personnel politique (par élection ou par nomination) et les priorités des élus. Si les questions discutées correspondent aux priorités des dirigeants en place et qu'elles appartiennent aux idées qui sont dans l'esprit du temps, les chances sont grandes qu'une "fenêtre politique » s'ouvre. Examinons le dossier de sécurité alimentaire à la lumière de ce qui précède.

Selon Kingdon (1995), un problème retient l'attention lorsqu'une crise ou un événement particulier survient. II arrive également que le suivi d'indicateurs systématiques, ou le suivi de programmes existants, contribue à dégager des situations problématiques. C'est le cas ici. Le problème de l'alimentation dans les milieux défavorisés est dans le paysage politique depuis longtemps. Le CQRS subventionne des projets qui s'intéressent à l'action des organismes communautaires offrant de l'aide alimentaire. II n'y a qu'un pas à faire pour élargir la problématique à celle, latente, de l'insécurité alimentaire. Des données précises sur l'insécurité alimentaire venues du milieu de la recherche et de l'enquête sur les populations en fournissent l'occasion.

Dans le courant des solutions, les idées et les connaissances circulent. Par un processus d'accumulation des connaissances, les spécialistes d'un secteur en viennent à formuler des propositions de solutions, des projets de politiques. Ceux-ci sont repris par les politiciens, les groupes d'intérêts, les bureaucrates, etc. Dans le cas qui nous intéresse, des solutions au problème d'insécurité 
alimentaire qui sont réalisables sur les plans social, économique et politique existent. Bien qu'elles soient très partielles, ces solutions sont perçues comme pouvant contribuer à la lutte contre la pauvreté : elles pourraient notamment « aider à briser le cycle intergénérationnel de la pauvreté et concourir de ce fait à sa prévention ». Les entrepreneurs sont nombreux. Les premiers efforts sont venus de la base il y a une dizaine d'années : ils sont le fait de la détermination des acteurs locaux et régionaux qui, avec le temps, se sont organisés en réseau et ont réalisé des projets en sécurité alimentaire. Ce sont eux qui, par une démarche de démonstration étalée sur une décennie, ont montré qu'il y avait des solutions " alternatives » à l'aide alimentaire. En plus des organismes de la communauté et des RRSSS, il y a également le MSSS, le CQRS et les chercheurs de divers domaines (développement communautaire; géographie; nutrition; service social; sociologie) qui ont agi en partenariat avec la communauté. Le plaidoyer prononcé par un membre du comité consultatif formé par le MESS semble avoir donné de la valeur à l'idée de la sécurité alimentaire.

Pour qu'une proposition de solution dure dans le temps, elle doit satisfaire certains critères, dont la plausibilité sur le plan technique, la correspondance aux valeurs dominantes, les considérations budgétaires et l'approbation par le public en général. L'axe d'intervention en sécurité alimentaire, proposé à la suite du plaidoyer, satisfaisait les trois premiers critères; la probabilité qu'il réponde au quatrième était grande.

Les changements qui surviennent dans le climat politique ou l'opinion publique, de même que les résultats électoraux, ont des répercussions notables sur l'agenda gouvernemental à moyen terme. Dans le cas à l'étude, le courant de la politique est présent: la priorité accordée par le gouvernement québécois à la lutte contre la pauvreté a permis aux acteurs intéressés par la question de la sécurité alimentaire d'inscrire l'objet de leurs préoccupations à l'agenda. II n'y a pas si longtemps encore, les chances de voir ce thème inscrit à l'agenda étaient plutôt minces, pour ne pas dire inexistantes (Lemieux, 1997) : non seulement le problème de l'insécurité alimentaire n'était pas suffisamment circonscrit, mais le contexte politique n'était pas favorable. 
Cependant, lorsque s'amorcent les travaux entourant l'élaboration d'une stratégie gouvernementale de lutte à la pauvreté, la situation est différente. D'une part, le problème de l'insécurité alimentaire est mieux défini. D'autre part, des entrepreneurs du dossier de la sécurité alimentaire se retrouvent activement engagés dans le processus d'élaboration de cette stratégie, tant au sein du MESS qu'au sein du comité consultatif formé par ce ministère. Ces entrepreneurs réussissent alors à dégager la relation entre l'insécurité alimentaire et la pauvreté au point de convaincre le MESS que la sécurité alimentaire doit s'insérer dans la stratégie gouvernementale de lutte contre la pauvreté. À cet égard, le plaidoyer prononcé par l'un des membres du comité consultatif, en avril 2001, semble avoir rejoint la chaîne d'influence au sein de l'appareil gouvernemental. Ce plaidoyer arrive en effet au terme d'un long processus d'accumulation de connaissances sur la question de la sécurité alimentaire. II survient au moment où le MESS est à la recherche de moyens novateurs pour lutter contre la pauvreté. La nature des arguments avancés dans le plaidoyer est compatible avec les buts et les intérêts du Ministère. La prise de conscience s'élargit au fur et à mesure qu'on chemine vers la stratégie.

En somme, les travaux entourant l'élaboration de la stratégie ont fourni une occasion privilégiée aux acteurs convaincus de l'importance de la sécurité alimentaire de faire inscrire cette question à l'agenda décisionnel. Comme l'explique Kingdon (1995), il arrive parfois, par un " effet de contagion », que l'ouverture d'une fenêtre sur un sujet favorise l'ouverture d'une autre fenêtre sur un sujet analogue ou connexe. Ainsi, la mise à jour de cette grande politique " englobante » de lutte contre la pauvreté, dont l'approche comportait déjà un angle nutritionnel (soutien alimentaire dans les écoles), aurait constitué une "fenêtre politique » dont se sont saisis les entrepreneurs pour porter avec succès la question de la sécurité alimentaire jusqu'au niveau décisionnel. C'est ainsi que le thème de la sécurité alimentaire est présent dans l'Énoncé de politique de la Stratégie nationale de lutte contre la pauvreté et l'exclusion sociale que le gouvernement s'apprête à inscrire dans une loi (ministère de l'Emploi et de la Solidarité sociale, 2002a). II 
paraît aussi au " Bilan" " des actions gouvernementales en la matière (ministère de l'Emploi et de la Solidarité sociale, 2002b). Malgré tout, la sécurité alimentaire n'est pas explicitement incluse dans le projet de loi $n^{\circ} 112$ visant à lutter contre la pauvreté et l'exclusion sociale, présenté le 12 juin 2002 par la ministre de la Solidarité sociale. Cependant, à l'automne 2002, dans le cadre des consultations ad hoc de la Commission des affaires sociales, l'action conjuguée de plusieurs entrepreneurs de la société civile (p. ex. : Association québécoise des banques alimentaires et des moissons, 2002; Collectif pour une loi sur l'élimination de la pauvreté, 2002; Front commun des personnes assistées sociales du Québec, 2002; Regroupement des cuisines collectives du Québec, 2002) est venue appuyer l'à-propos de la couverture des besoins essentiels, dont la sécurité alimentaire. II en résultera un amendement au projet de loi 112. L'objet de la sécurité alimentaire devient matière de responsabilité publique lorsqu'il est finalement inscrit à l'article $9^{10}$ du projet de loi soumis pour adoption par l'Assemblée nationale du Québec. La Loi visant à lutter contre la pauvreté et l'exclusion sociale est sanctionnée le 18 décembre 2002. Les dispositions de la loi liées à l'article 9 entrent en vigueur le 5 mars 2003.

\section{CONCLUSION}

Par son caractère potentiellement omniprésent, voire lancinant, l'insécurité alimentaire est l'une des dimensions les plus tangibles de la pauvreté. Paradoxalement, il s'agit d'un phénomène qu'on a tendance à occulter dans nos sociétés d'abondance. Avec un investissement pour des projets en matière de sécurité alimentaire, l'initiative du Québec marque-elle le début du renversement de

9. Dans le bilan gouvernemental, les activités de soutien alimentaire et les projets de type alternatif en sécurité alimentaire sont fondus sous le vocable " sécurité alimentaire », auquel on attribue un budget global de l'ordre de 35 millions de dollars.

10. Le passage de l'article 9 , qui se rapporte à la sécurité alimentaire, se lit comme suit : "Les actions liées au renforcement du filet de sécurité sociale et économique doivent notamment viser à : ... $4^{\circ}$ favoriser, pour les personnes et les familles en situation de pauvreté, l'accès, en toute dignité, à un approvisionnement alimentaire suffisant et nutritif, à un coût raisonnable, de même qu'à une information simple et fiable qui leur permette de faire des choix alimentaires éclairés; ... » 
cette tendance? Bien qu'il s'agisse d'une mesure modeste et non récurrente, nous voyons là une ouverture signifiante. Le thème de la sécurité alimentaire des ménages est inscrit en bonne et due forme dans la loi 112. Cette ouverture a bénéficié d'un " effet de contagion » qui a pu se réaliser pleinement par l'action convergente des trois courants précités et des entrepreneurs qui y ont pris part.

Les quelque cinquante participants au colloque « L'action communautaire et les politiques pour la sécurité alimentaire: une question sociale et de santé » (Delisle et Hamelin, 1997) avaient posé l'hypothèse que la progression du dossier de l'insécurité alimentaire passe par l'action politique.

À l'issue de cette étude, quatre actions fondamentales apparaissent susceptibles de concourir à la réduction de la pauvreté :

1. Revoir la répartition financière gouvernementale entre " soutien alimentaire " et " sécurité alimentaire ». Le premier pouvant être considéré comme un cataplasme utile, certes, mais largement insuffisant à moyen terme pour favoriser l'autonomie des personnes.

2. Utiliser la sécurité alimentaire comme indicateur de développement social. Cela exige au préalable la construction d'indicateurs fiables de sécurité alimentaire.

3. Évaluer l'impact des projets mis en œuvre dans le cadre de la Stratégie sur la sécurité alimentaire des personnes, et aussi sur le développement social.

4. Pousser plus loin le dossier de l'alimentation sur l'échiquier politique, en vue d'une politique publique de sécurité nutritionnelle.

Les auteures remercient de leurs commentaires monsieur Vincent Lemieux, mesdames Suzie Lapointe et Anne O'Sullivan, de même que madame Lise Bertrand. 


\section{RÉFÉRENCES BIBLIOGRAPHIQUES}

ANDERSON, S.A. (1990). " Core Indicators of Nutritional State for Difficult to Sample Populations », Journal of Nutrition, 120, p. 1559-1600.

ASSOCIATION CANADIENNE DE LA JOURNÉE MONDIALE DE L'ALIMENTATION (1992). Plan stratégique 1993-1996, Ottawa, L'Association, 19 p.

ASSOCIATION QUÉBÉCOISE DES BANQUES ALIMENTAIRES ET DES MOISSONS (2002). Exposé devant la Commission des affaires sociales, 5 novembre. Québec, Les travaux parlementaires de la $36^{\mathrm{e}}$ législature, $2^{\mathrm{e}}$ session, Journal des débats. Consultation sur Internet.

BeEman, J., J. PAnet-Raymond et J. Rouffignat (1996). Du dépannage alimentaire au développement communautaire, Guide d'animation, Université de Montréal, École de service social, 84 p.

BERTRAND, L. et M. DUPONT (1998). Évaluation du projet régional de développement de la sécurité alimentaire dans la région de Montréal-Centre, Régie régionale de la santé et des services sociaux de Montréal-Centre, Direction de la santé publique, $91 \mathrm{p}$.

CAmpBell, C. (1991). «Food Insecurity: A Nutritional Outcome or a Predictor Variable? », Journal of Nutrition, 121, p. 408-415.

CENTRALE DES SYNDICATS DÉMOCRATIQUes (2000). " L'élimination de la pauvreté est une condition de la réalisation de l'égalité des droits! », La Centrale des syndicats démocratiques: un acteur social engagé. Consultation sur Internet.

CobB, R.W. et C.D. Elder (1983). Participation in American Politics : The Dynamics of Agenda-building, Baltimore, Johns Hopkins University Press, 196 p.

ColiN, C. (1997). "Sécurité alimentaire ». Discours prononcé dans le cadre du lancement du Mois de la nutrition 1997 à l'Institut du tourisme et de l'hôtellerie du Québec, Montréal, le 25 février.

Collectif POUR UNE LOI SUR L'ÉLIMINATION DE LA PAUVRETÉ (2000). Plus de 215000 signatures pour une loi sur l'élimination de la pauvreté, Québec. Communiqué du 22 novembre. 
COLLECTIF POUR UNE LOI SUR L'ÉLIMINATION DE LA PAUVRETÉ (2002). Exposé devant la Commission des affaires sociales, $1^{\text {er }}$ octobre, Québec. Les travaux parlementaires de la $36^{\mathrm{e}}$ législature, $2^{\mathrm{e}}$ session, Journal des débats. Consultation sur Internet.

CONSEIL DE LA SANTÉ ET DU BIEN-ÊTRE SOCIAL (1998). Forum sur le développement social : rapport remis au ministre de la Santé et des Services sociaux, Québec, Le Conseil, 139 p.

CôtÉ, E., S. RACINE et J. Rouffignat (1995). « Appauvrissement, aide alimentaire et organismes communautaires. De la compréhension à l'action » Service social, vol. 44, nº 3, p. 95-114.

Delisle, H. et A.-M. HAmelin (dir.) (1997). L'action communautaire et les politiques pour la sécurité alimentaire, une question sociale et de santé, Actes du Colloque, $64^{\mathrm{e}}$ Congrès de l'ACFAS, Les cahiers scientifiques (91), Montréal, ACFAS, 118 p.

DufouR, R. (1992). Au-delà du dépannage alimentaire. Document de travail pour le colloque, Québec, Comité Québec de la Journée mondiale de l'alimentation. $21 \mathrm{p}$.

FÉdÉRATION DES FEMMES DU QUÉBEC (1995). La marche « Du pain et des roses $»$. Consultation sur Internet.

FRONT COMMUN DES PERSONNES ASSISTÉES SOCIALES DU QUÉBEC (2002). Exposé devant la Commission des affaires sociales, 30 octobre. Québec, Les travaux parlementaires de la $36^{\mathrm{e}}$ législature, $2^{\mathrm{e}}$ session, Journal des débats. Consultation sur Internet.

GoUVERNEMENT DU QUÉBEC (2001). Québec intensifie son action en matière de lutte contre la pauvreté. Communiqué du 15 juin.

HAMELIN, A.-M. (1999). L'insécurité alimentaire des ménages dans la région de Québec : une exploration. Thèse de doctorat, Québec, Université Laval, 254 p.

Hamelin, A.-M., M. BeAudRY et J.-P. HABICHT (2002). " Characterization of Household Food Insecurity in Québec : Food and Feelings ", Social Science and Medicine, 54, p. 119-132.

HAMELIN, A.-M., J.-P. HABICHT et M. BeAudRY (1999). « Food Insecurity : Consequences for the Household and Broader Social Implications », Journal of Nutrition, 129, p. 525S-528S. 
INSTITUT DE LA STATISTIQUE DU QUÉBEC (2000). Enquête sociale et de santé 1998, chap. 6, Collection La santé et le bien-être, Québec, Institut de la statistique du Québec.

Kingdon, J.W. (1995). Agendas, Alternatives and Public Policies, New York, Harper Collins College Publishers, 253 p.

Leduc-Gauvin, J., M. Cossette, L. Lépine et M. Malette (1996). Agir ensemble pour contrer l'insécurité alimentaire, Montréal, Ordre professionnel des diététistes du Québec, $30 \mathrm{p}$.

LeMiEUX, L. (1995). Projet pilote «Pour une approche globale d'intégration sociale et professionnelle des prestataires de la sécurité du revenu : soutien à l'alimentation pour les familles monoparentales prestataires de la sécurité du revenu », Québec, le ministère de la Main-d'œuvre, de la Sécurité du revenu et de la Formation professionnelle.

LemieuX, V. (1997). "Conditions à l'adoption de politiques de sécurité alimentaire », dans H. Delisle et A.-M. Hamelin (dir.), L'action communautaire et les politiques pour la sécurité alimentaire, une question de santé. Actes du Colloque, $64^{\mathrm{e}}$ Congrès de l'ACFAS, Les cahiers scientifiques (91), Montréal, ACFAS, p. 75-81.

LES PARTENAIRES POUR LE DÉVELOPPEMENT DE LA SÉCURITÉ ALIMENTAIRE (2000). "Sortir de l'impasse : comprendre et agir au-delà de la faim, colloque du 9 et 10 novembre 1999 », Des gestes plus grands que la panse, dossier 3, Montréal, Direction de la santé publique de Montréal-Centre, $8 \mathrm{p}$.

LOI VISANT À LUTTER CONTRE LA PAUVRETÉ ET L'EXCLUSION SOCIALE. Lois du Québec 2002, c. 61.

Ministère dE L'EMPLOI ET DE LA SolidARItÉ SOCIALE (2001). Ne laisser personne de côté. Orientations et perspectives d'action en matière de lutte contre la pauvreté, Québec, Le Ministère, 35 p.

MINISTÈRE DE L'EMPLOI ET DE LA SOLIDARITÉ SOCIALE (2002a). La volonté d'agir, la force de réussir. Stratégie nationale de lutte contre la pauvreté et l'exclusion sociale, Énoncé de politique, Québec, Le Ministère, $57 \mathrm{p}$. 
MINISTĖRE DE L'EMPLOI ET DE LA SOLIDARITÉ SOCIALE (2002b). La volonté d'agir, la force de réussir. Stratégie nationale de lutte contre la pauvreté et l'exclusion sociale. Bilan, Québec, Le Ministère, 2 p.

Ministère de LA SANTÉ ET DES SeRVICES SociauX (1992). La politique de la santé et du bien-être, Québec, Le Ministère, $192 \mathrm{p}$.

Ministère de LA SANTÉ ET DES SeRVICES SociauX (1993). Un appel à l'action en nutrition au Québec. Actes du colloque tenu les 28 et 29 avril 1993 à Montréal, Québec, Le Ministère, 126 p.

Ministère de La SANTÉ et des SeRVICES SociauX (1997). Priorités nationales de santé publique 1997-2002, Québec, Le Ministère, $103 \mathrm{p}$.

Ministère de LA SANTÉ ET DES SeRVICES Sociaux (2002). Cadre de référence. Programme de subvention de projet en matière de sécurité alimentaire, 2002-2004, Québec, Le Ministère, 10 p.

MinistèRE DES FINANCES (2001). Discours sur le budget. Budget 20012002. Prononcé à l'Assemblée nationale par madame Pauline Marois, le 29 mars.

PORTZ, J. (1996). «Problem Definitions and Policy Agendas: Shaping the Educational Agenda in Boston », Policy Studies Journal, vol. 24, p. 371-386.

Radimer, K.L., C.M. Olson, J.C. Greene, C.C. Campbell et J.-P. HABICHT (1992). « Understanding Hunger and Developing Indicators to Assess It in Women and Children », Journal of Nutrition Education, 24, p. 36S-45S.

RegrouPEMENT DES CUISINES COLLECTIVES DU QuÉBEC (2002). Exposé devant la Commission des affaires sociales, 24 octobre. Québec, Les travaux parlementaires de la $36^{\mathrm{e}}$ législature, $2^{\mathrm{e}}$ session, Journal des débats. Consultation sur Internet.

Rouffignat, J., S. RACINE et E. CôtÉ (1996). Appauvrissement, aide alimentaire et organismes communautaires: de la compréhension à l'action. Rapport de recherche, Québec, CRAD, 265 p. 
Rouffignat, J., L. Dubois, J. PANet-RAymond, P. LAMONTAGne, S. CAMERON et M. GIRARD (2001). De la sécurité alimentaire au développement social, les effets des pratiques alternatives dans les régions du Québec 1999-2000. Rapport synthèse, Québec, Université Laval, Département de géographie, $181 \mathrm{p}$.

SABOURIN, P., R. HuRtubise et J. LACOURSe (2000). Citoyens, bénéficiaires et exclus - Usages sociaux et modes de distribution de l'aide alimentaire dans deux régions du Québec: la Mauricie et l'Estrie, Montréal, Sherbrooke et Québec, Université de Montréal, Université de Sherbrooke et Conseil québécois de la recherche sociale, $365 \mathrm{p}$.

SoROKA, S.N. (2002) Agenda-setting Dynamics in Canada, Vancouver, UBC Press, $168 p$.

STATISTIQUE CANADA (2001). "L'insécurité alimentaire dans les ménages canadiens », Le Quotidien, mercredi 15 août.

TABLE DE CONCERTATION SUR LA FAIM DU MONTRÉAL MÉTROPOLITAIN (1992). Colloque « La faim c'est assez, le dépannage ce n'est pas assez », Montréal.

TABLE DE CONCERTATION SUR LA FAIM DU MONTRÉAL MÉTROPOLITAIN (1993). Répertoire des organismes d'aide alimentaire du Montréal métropolitain, Montréal, La Table. 294 p.

TABLE DE CONCERTATION SUR LA FAIM DU MONTRÉAL MÉTROPOLITAIN (1996). Colloque «Faim et pouvoir : l'urgence d'entrer dans une dynamique de sécurité alimentaire, » Montréal, Université du Québec à Montréal, 7 et 8 novembre.

TABLE D'INTERACTION SUR LA FAIM DE QUÉBEC (1994). L'aide alimentaire : construire des dynamiques alternatives au problème de la faim. Actes du colloque, Québec, La Table, 37 p.

UNITED NATIONS - ACC/SNC (1991) Some options for improving nutrition in the 1990s, Supplement to SNC News, 7, p. 5, 19 p.

United States Department of Agriculture (USDA) (2000). Household Food Security in the United States, 1999, Washington (DC), Economic Research Service, Food Assistance and Nutrition Research Report No. 8. 